\title{
Diffuse PeV neutrino emission from Ultra-Luminous Infrared Galaxies
}

\author{
Hao-Ning He, ${ }^{1}$ Tao Wang, ${ }^{2,3}$ Yi-Zhong Fan*, ${ }^{1}$ Si-Ming Liu, ${ }^{1}$ and Da-Ming Wei ${ }^{1}$ \\ ${ }^{1}$ Key Laboratory of Dark Matter and Space Astronomy, \\ Purple Mountain Observatory, Chinese Academy of Sciences, Nanjing 210008, China \\ ${ }^{2}$ School of Astronomy and Space Science, Nanjing University, Nanjing, 210093, China \\ ${ }^{3}$ Key laboratory of Modern Astronomy and Astrophysics(Nanjing University), Ministry of Education, Nanjing 210093, China
}

\begin{abstract}
Ultra-luminous infrared galaxies (ULIRGs) are the most luminous and intense starburst galaxies in the Universe. Both their star-formation rate (SFR) and gas surface mass density are very high, implying a high supernovae rate and an efficient energy conversion of energetic protons. A small fraction of these supernovae is the so-called hypernovae with a typical kinetic energy $\sim 10^{52}$ erg and a shock velocity $\geq 10^{9} \mathrm{~cm} \mathrm{~s}^{-1}$. The strong shocks driven by hypernovae are able to accelerate cosmic ray protons up to $10^{17} \mathrm{eV}$. These energetic protons lose a good fraction of their energy through proton-proton collision when ejected into very dense interstellar medium, and as a result, produce high energy neutrinos $(\leq 5 \mathrm{PeV})$. Recent deep infrared surveys provide solid constraints on the number density of ULIRGs across a wide redshift range $0 \leqslant z \leqslant 2.3$, allowing us to derive the flux of diffuse neutrinos from hypernovae. We find that at $\mathrm{PeV}$ energies, the diffuse neutrinos contributed by ULIRGs are comparable with the atmosphere neutrinos with the flux of $2 \times 10^{-9} \mathrm{GeVcm}^{-2} \mathrm{~s}^{-1} \mathrm{sr}^{-1}$, by assuming the injected cosmic ray spectrum as $d N_{\mathrm{p}}^{\prime} / d \varepsilon_{\mathrm{p}}^{\prime} \propto \varepsilon_{\mathrm{p}}^{\prime-2}$.
\end{abstract}

PACS numbers: 95.85.Ry, 95.85.Hp, 98.70.Sa

\section{INTRODUCTION}

The galactic supernova remnants (SNRs) are widely suggested to be the dominant source for the cosmic rays (CRs) at energies below the "knee" at $\sim 3 \times 10^{15} \mathrm{eV}$, most probably through the diffusive shock acceleration mechanism [1]. Though the details are still to be figured out, it is generally believed that the maximum energy of CRs accelerated by SNRs depends on both the velocity and the kinetic energy of the supernova outflow. A small fraction of the supernovae has a typical kinetic energy $E_{\mathrm{k}} \sim 0.5-5 \times 10^{52} \mathrm{erg}$ and a typical velocity $V \sim 10^{9} \mathrm{~cm} \mathrm{~s}^{-1}\left(E_{\mathrm{k}} / 10^{52} \mathrm{erg}\right)^{1 / 2}\left(M_{\mathrm{SN}} / 10 M_{\odot}\right)^{-1 / 2}$, both are substantially larger than that of normal supernova, where $M_{\mathrm{SN}}$ is the rest mass of the SN ejecta. These peculiar supernovae, such as SN 1997ef, SN 1997dq, SN 1998bw and SN 2002ap, have been called the hypernovae [2 4]. The maximum energy of the protons accelerated at the shock front of a supernova expanding into the uniform dense interstellar medium (ISM) can be estimated as $\varepsilon_{\mathrm{p}, \max }^{\prime} \approx 10^{17} \mathrm{eV}\left(\frac{V}{10^{9} \mathrm{~cm} \mathrm{~s}^{-1}}\right)^{2}\left(\frac{n}{10^{3} \mathrm{~cm}^{-3}}\right)^{1 / 6}\left(\frac{M_{\mathrm{SN}}}{10 M_{\odot}}\right)^{1 / 3}$, where $n$ is the number density of ISM [5]. Such a fact motivates some colleagues to suggest that hypernova remnants are the dominant source of cosmic rays above the knee and the cosmic ray spectrum/flux up to $\sim 10^{18}-10^{19} \mathrm{eV}$ may be accounted for as long as the variety of supernovae has been taken into account [6 -10].

Ultra-luminous infrared galaxies (ULIRGs), first discovered in large numbers by the Infrared Astronomical Satellite in 1983, are among the most luminous objects in the local universe with infrared luminosity $L_{8-1000 \mu \mathrm{m}}>$ $10^{12} L_{\odot}[11,12]$. The large infrared luminosities are attributed to large amounts of dust, which absorb ultraviolet (UV) photons and re-radiate them in the infrared (IR). Comprehensive observations show that ULIRGs are powered mainly by a large population of hot young stars, i.e., a "starburst", with a significant fraction also containing an IR-luminous AGN [13]. Local ULIRGs are exclusively mergers of gas rich galaxies accompanied by concentrated dust-enshrouded starburst, with very high star formation rate $(\mathrm{SFR}) \gtrsim 200 \mathrm{M}_{\odot} \mathrm{yr}^{-1}$. The supernova rate is expected to be high and huge amounts of cosmic ray particles are accelerated. Moreover, the relatively small sizes $\sim 1 \mathrm{kpc}$ of ULIRGs mean that the ISM density is also quite high, i.e., $<n>\simeq 10^{3}-10^{4} \mathrm{~cm}^{-3}[14]$, suggesting that the accelerated high energy protons produced in ULIRGs have a great chance to interact with the interstellar medium nucleons, to produce pions, and decay into secondary electrons and positrons, $\gamma$-rays, and neutrinos. If the energy loss time of the protons through the proton-proton collisions is shorter than the starburst lifetime and the confinement time, the protons will lose most of their energy before escaping and produce interesting observational signals [15, 16].

Previously the possible high energy neutrino emission from the starburst galaxies has been investigated by assuming that the observed $\mathrm{GeV}$ photons are produced via the decay of pions [17, 18]. The events are expected to be significantly enhanced if the nearby starburst galaxies host some Gamma-ray Bursts (GRBs) [19]. In this work, we focus on the $\mathrm{PeV}$ neutrino emission produced via the interaction of the accelerated Cosmic Rays with the dense environment in the ULIRGs.

\section{THE HYPERNOVAE RATE IN THE ULIRGS}

Though ULIRGs are very rare in the local universe, they are vastly more numerous at high redshifts. The relative contribution of ULIRGs to the SFR density of the universe also increases with redshift, and may even be the dominant component at $z \sim 2[20]$. Thus it is essential 
to study the role of ULIRGs across a wide redshift range in producing $\mathrm{PeV}$ neutrinos. Observational data shows that core collapse supernovae of type SN-Ib/c contribute with $11 \%$ of the total $\mathrm{SN}$ rate 21]. The hypernovae rate to the normal $\mathrm{Ib} / \mathrm{c} \mathrm{SN}$ rate is $7 \%$ in the local universe [22]. Therefore the ratio of the hypernovae rate to the supernovae rate can be estimated to be $f_{\mathrm{HS}} \simeq 0.01$.

Recent IR observations show that the SFR density for ULIRGs increases rapidly at $z<1$, and stays as a constant at higher redshift $1.2 \leqslant z \leqslant 2.3$, which can be approximated as [23]

$$
\rho_{\mathrm{SFR}}(z)=\left\{\begin{array}{lr}
1.9 \times 10^{-4} e^{3.3 z} \mathrm{M}_{\odot} \mathrm{yr}^{-1} \mathrm{Mpc}^{-3}, \quad z<1.2 \\
0.01 \mathrm{M}_{\odot} \mathrm{yr}^{-1} \mathrm{Mpc}^{-3}, & 1.2 \leqslant z \leqslant 2.3 .
\end{array}\right.
$$

The supernova rate is related to the SFR via [24]

$$
R_{\mathrm{SN}}(z)=1.2 \times 10^{-2} \rho_{\mathrm{SFR}}(z) / M_{\odot} .
$$

Therefore, the hypernovae rate is

$$
\begin{aligned}
R_{\mathrm{HN}}(z) & =f_{\mathrm{HS}} R_{\mathrm{SN}}(z) \\
& =\left\{\begin{array}{l}
2.4 \times 10^{-8} e^{3.3 z} \mathrm{yr}^{-1} \mathrm{Mpc}^{-3}, \quad z<1.2 ; \\
1.2 \times 10^{-6} \mathrm{yr}^{-1} \mathrm{Mpc}^{-3}, \quad 1.2 \leqslant z \leqslant 2.3 .
\end{array}\right.
\end{aligned}
$$

Taking into account cosmology, the hypernovae number occurring in ULIRGs with redshift $\leqslant z$ per year is numerically calculated by

$$
N_{\mathrm{HN}}(z)=\frac{c}{H_{0}} \int_{0}^{z} d z \frac{R_{\mathrm{HN}}(z) 4 \pi D_{\mathrm{c}}(z)^{2}}{\sqrt{\Omega_{\Lambda}+(1+z)^{3} \Omega_{\mathrm{m}}}},
$$

where $D_{c}(z)=\frac{c}{H_{0}} \int_{0}^{z} d z \frac{1}{\sqrt{\Omega_{\Lambda}+(1+z)^{3} \Omega_{\mathrm{m}}}}$ is the comoving distance, $c$ is the light speed. Hereafter, we adopt the Hubble constant as $H_{0}=70 \mathrm{kms}^{-1} \mathrm{Mpc}^{-1}$, the matter density as $\Omega_{\mathrm{m}}=0.3$, and the dark energy density as $\Omega_{\Lambda}=0.7$ in the flat universe.

\section{DIFFUSE PEV NEUTRINO EMISSION FROM ULIRGS.}

The protons accelerated by the hypernovae would lose energy into the $\gamma$-ray photons, electrons and positrons, and neutrinos, through proton-proton collisions when injected into the interstellar mediums. A part of the protons energy will convert into neutrinos via the decay of charged pions, $\pi^{+} \rightarrow \mu^{+}+\nu_{\mu} \rightarrow e^{+}+\nu_{e}+\bar{\nu}_{\mu}+\nu_{\mu}$ and $\pi^{-} \rightarrow \mu^{-}+\bar{\nu}_{\mu} \rightarrow e^{-}+\bar{\nu}_{e}+\bar{\nu}_{\mu}+\nu_{\mu}$.

The energy loss time of protons is $\tau_{\text {loss }}=\left(0.5 n \sigma_{\mathrm{pp}} c\right)^{-1}$, where the factor 0.5 is inelasticity [25], and $\sigma_{\mathrm{pp}}$ is the inelastic nuclear collision cross section, which is $\gtrsim 70 \mathrm{mb}$ for the protons at energies $\varepsilon_{\mathrm{p}}^{\prime} \geq 10 \mathrm{PeV}$ that is of our great interest. Introducing a parameter $\Sigma_{\text {gas }} \equiv m_{\mathrm{p}} n l$ as the surface mass density of the gas, with $l$ as the scale of the dense region in the galaxies, the energy loss time reads [26]

$$
\tau_{\text {loss }}=1.4 \times 10^{4} \mathrm{yr} \frac{l}{100 \mathrm{pc}}\left(\frac{\Sigma_{\mathrm{gas}}}{1.0 \mathrm{~g} \mathrm{~cm}^{-2}}\right)^{-1} .
$$

Gas surface density can be derived from their SFR density based on an empirical relation that SFR surface density scales as some positive power $\beta \sim 1.4$ of the local gas surface density, i.e., the Kennicutt-Schmidt law [27].

$$
\Sigma_{\mathrm{SFR}}=(2.5 \pm 0.7) \times 10^{-4}\left(\frac{\Sigma_{\text {gas }}}{1 \mathrm{M}_{\odot} \mathrm{pc}^{-2}}\right)^{1.4 \pm 0.15} \mathrm{M}_{\odot} \mathrm{yr}^{-1} \mathrm{kpc}^{-2} .
$$

ULIRGs have SFR $\gtrsim 200 M_{\odot} \mathrm{yr}^{-1}[28]$. If we assume a half-light radius of $\sim 1 \mathrm{kpc}$, it yields a SFR density $\gtrsim 32 M_{\odot} \mathrm{yr}^{-1} \mathrm{kpc}^{-2}$, consistent with observations of ULIRGs at both low and high redshifts. According to the Kennicutt-Schmidt law, the derived gas surface density is $\gtrsim 1.0 \mathrm{~g} \mathrm{~cm}^{-2}$. Hence, the energy loss time for the known ULIRGs is much shorter than the starburst lifetime in ULIRGs, which ranges from $10^{7}$ to $10^{8}$ years [29].

Another important factor to determine the fraction of protons energy conversion is the magnetic confinement time of protons. Adopting the similar confinement time as the Galaxy, and simply considering that the confinement time depends on the proton's Larmor radius $\left(\propto \varepsilon_{\mathrm{p}}^{\prime} / B\right)$, the confinement time of the proton with energy $\varepsilon_{\mathrm{p}}^{\prime}$ can be estimated as [17]

$$
\tau_{\text {conf }} \approx 10^{4} \mathrm{yr}\left(\frac{\varepsilon_{\mathrm{p}}^{\prime}}{10 \mathrm{PeV}}\right)^{-0.5}\left(\frac{B}{3 \mu \mathrm{G}}\right)^{0.5} .
$$

It is found in Thompson et al. (2006) [30] that the magnetic field strength of the starburst galaxies scales linearly with $\Sigma_{\text {gas }}$, i.e., $B \approx 3 \mu \mathrm{G} \frac{\Sigma_{\text {gas }}}{2.5 \times 10^{-3} \mathrm{gcm}^{-2}}$. Consequently, the confinement time can be rewritten as

$$
\tau_{\text {conf }} \approx 2 \times 10^{5} \mathrm{yr}\left(\frac{\varepsilon_{\mathrm{p}}^{\prime}}{10 \mathrm{PeV}}\right)^{-0.5}\left(\frac{\Sigma_{\mathrm{gas}}}{1.0 \mathrm{gcm}^{-2}}\right)^{0.5} .
$$

The fraction of the energy that the protons lose into pions is $f_{\pi}=1-\exp \left(-\tau_{\text {conf }} / \tau_{\text {loss }}\right)$, which is close to 1 as long as $\tau_{\text {conf }} \geqslant \tau_{\text {loss }}$. As a result, the protons with energy $\varepsilon_{\mathrm{p}}^{\prime}$ lose almost all of their energy via the proton-proton collision before escaping from the starburst galaxies as long as $\tau_{\text {loss }} \leq \tau_{\text {conf }}$, which constrains the critical gas surface density $\Sigma_{\text {crit }}$ as

$$
\Sigma_{\text {gas }} \gtrsim \Sigma_{\text {crit }}=0.17 \mathrm{~g} \mathrm{~cm}^{-2}\left(\frac{\varepsilon_{\mathrm{p}}^{\prime}}{10 \mathrm{PeV}}\right)^{1 / 3}\left(\frac{l}{100 \mathrm{pc}}\right)^{2 / 3} .
$$

Consequently, for the ULIRGs with the gas surface density $\Sigma_{\text {gas }} \gtrsim 1.0 \mathrm{~g} \mathrm{~cm}^{-2}$, the CR protons with energy up to $\sim 2.0 \times 10^{3} \mathrm{PeV}\left(\frac{l}{100 \mathrm{pc}}\right)^{-2}$ will lose almost all their energy via interacting with the dense ISM.

The charged pion, whose energy is $\varepsilon_{\pi}^{\prime}=0.2 \varepsilon_{p}^{\prime}$, will then decay to produce four leptons, which share the energy equally. Therefore, the fraction of the protons energy converted into neutrinos is $\epsilon_{\nu}=0.05$ [31]. The observed neutrinos in the energy range $\left(\varepsilon_{\nu, 1}, \varepsilon_{\nu, 2}\right)$ are produced by the protons in the energy range $\left(\varepsilon_{p, 1}^{\prime}, \varepsilon_{p, 2}^{\prime}\right)$ 
in the ULIRGs, where $\varepsilon_{p, 1}^{\prime}=(1+z) \varepsilon_{\nu, 1} / \epsilon_{\nu}$ and $\varepsilon_{p, 2}^{\prime}=$ $(1+z) \varepsilon_{\nu, 2} / \epsilon_{\nu}$. The energy fraction of the protons producing the neutrinos with energy between $\varepsilon_{\nu, 1}$ and $\varepsilon_{\nu, 2}$ is (for $\alpha>2.0$ )

$$
\epsilon_{\mathrm{dec}}^{\prime}=\frac{\varepsilon_{p, 1}^{\prime 2-\alpha}-\varepsilon_{p, 2}^{\prime 2-\alpha}}{\varepsilon_{p, \text { max }}^{\prime 2-\alpha}-\varepsilon_{p, \text { min }}^{\prime 2-\alpha}}=\left(\frac{1+z}{\epsilon_{\nu}}\right)^{2-\alpha} \epsilon_{\mathrm{dec}}
$$

where we assume the spectrum of the ejected protons as $\frac{d N_{p}^{\prime}}{d \varepsilon_{p}^{\prime}} \propto \varepsilon_{p}^{\prime-\alpha}$, and $\varepsilon_{\mathrm{p}, \text { min }}^{\prime} \sim 2 \mathrm{GeV}$ is the minimum energy of the ejected protons in the rest frame, and we define a parameter independent on the redshift, $\epsilon_{\mathrm{dec}} \equiv \frac{\varepsilon_{\nu, 1}^{2-\alpha}-\varepsilon_{\nu, 2}^{2-\alpha}}{\varepsilon_{p, \max }^{\prime 2-\alpha}-\varepsilon_{p, \min }^{2-\alpha}}$. Adopting an efficiency factor $\eta=0.05-0.15$ for the conversion of ejecta kinetic energy into the relativistic CR protons energy [1, 19], the total energy of the CR protons is $E_{\mathrm{CR}}=\eta E_{\mathrm{HN}}$. Hereafter, we take the typical kinetic energy of the hypernova as $E_{\mathrm{HN}}=2 \times 10^{52} \mathrm{erg}$ and $\eta=0.1$. Adopting $\alpha \sim 2.1$, for the neutrinos with energies $0.5-5 \mathrm{PeV}$, we have $\epsilon_{\mathrm{dec}} \simeq 0.07$. The total energy of the observed neutrinos from $\varepsilon_{\nu, 1}$ to $\varepsilon_{\nu, 2}$ produced by each hyperonva in the ULIRGs is estimated as

$E_{\nu} \approx 4 \times 10^{48} \operatorname{erg} \frac{E_{\mathrm{HN}}}{2 \times 10^{52} \mathrm{erg}} \frac{\eta}{0.1} \frac{\epsilon_{\mathrm{dec}}}{0.07}\left(\frac{\epsilon_{\nu}}{0.05}\right)^{\alpha-1}\left(\frac{1+z}{3}\right)^{2-\alpha}$

The produced neutrinos have the similar spectrum as the ejected protons, i.e., the observed neutrinos spectrum is $\frac{d N_{\nu}}{d \varepsilon_{\nu}}=N_{\mathrm{c}} \varepsilon_{\nu}^{-\alpha}$ [31], then the normalized coefficient of the neutrino spectrum can be calculated via

$$
N_{\mathrm{c}}=\frac{E_{\nu}(2-\alpha)}{\varepsilon_{\nu, 2}^{2-\alpha}-\varepsilon_{\nu, 1}^{2-\alpha}}=A E_{\nu}
$$

where we define a parameter $A \equiv \frac{2-\alpha}{\varepsilon_{\nu, 2}^{2-\alpha}-\varepsilon_{\nu, 1}^{2-\alpha}}$ to simplify the expression. Consequently, the diffuse $\mathrm{PeV}$ neutrino flux integrating from local to the high redshift $z$ reads

$$
\begin{aligned}
F_{\nu}\left(\varepsilon_{\nu}\right)= & \left.\frac{1}{4 \pi} \frac{c}{H_{0}} \int_{0}^{z} d z \frac{4 \pi D_{\mathrm{c}}(z)^{2} R_{\mathrm{HN}}(z) N_{\mathrm{c}} \varepsilon_{\nu}^{2-\alpha}}{4 \pi D_{\mathrm{L}}(z)^{2} \sqrt{\Omega_{\mathrm{M}}(1+z)^{3}+\Omega_{\Lambda}}}\right) \\
= & \varepsilon_{\nu}^{2-\alpha} A \epsilon_{\mathrm{dec}} \eta \epsilon_{\nu}^{\alpha-1} E_{\mathrm{HN}} \\
& \times \frac{c}{H_{0}} \int_{0}^{z} d z \frac{R_{\mathrm{HN}}(z)}{(1+z)^{\alpha} \sqrt{\Omega_{\mathrm{M}}(1+z)^{3}+\Omega_{\Lambda}}}
\end{aligned}
$$

where the luminosity distance $D_{\mathrm{L}}=(1+z) D_{\mathrm{c}}$, while for the specified case with $\alpha=2$, it reads

$$
\begin{aligned}
F_{\nu}\left(\varepsilon_{\nu}\right)= & A^{*} \epsilon_{\mathrm{dec}}^{\prime *} \eta \epsilon_{\nu} E_{\mathrm{HN}} \\
& \times \frac{c}{H_{0}} \int_{0}^{z} d z \frac{R_{\mathrm{HN}}(z)}{(1+z)^{2} \sqrt{\Omega_{\mathrm{M}}(1+z)^{3}+\Omega_{\Lambda}}}
\end{aligned}
$$

with $A^{*}=\frac{1}{\ln \varepsilon_{\nu, 2}-\ln \varepsilon_{\nu, 1}}$ and $\epsilon_{\mathrm{dec}}^{\prime *}=\frac{\ln \varepsilon_{p, 2}^{\prime}-\ln \varepsilon_{p, 1}^{\prime}}{\ln \varepsilon_{p, \max }^{\prime}-\ln \varepsilon_{p, \min }^{\prime}}$.

In figure 1., we show the flux of the diffuse neutrinos, at the energy of $1 \mathrm{PeV}$, from ULIRGs, GRBs and AGNs, for the ejected protons spectrum $\frac{d N_{p}^{\prime}}{d \varepsilon_{p}^{\prime}} \propto \varepsilon_{p}^{\prime-2}$. The diffuse
$\mathrm{PeV}$ neutrino flux contributed by ULIRGs from local to $z=2.3$ is $F_{\nu, \mathrm{PeV}} \simeq 2 \times 10^{-9} \mathrm{GeV} \mathrm{cm}{ }^{-2} \mathrm{~s}^{-1} \mathrm{sr}^{-1}$ for $\alpha=2$, which is comparable with the flux of atmosphere $\mathrm{PeV}$ neutrinos, implying an un-ignorable contribution to the $\mathrm{PeV}$ neutrino flux. However, the detection prospect of $\mathrm{PeV}$ neutrinos from ULIRGs is not promising.

We estimate the amount of the detection rate of neutrinos in the energy range $\left(\varepsilon_{\nu, 1}, \varepsilon_{\nu, 2}\right)$ via

$$
\begin{aligned}
N_{\nu}= & A \epsilon_{\mathrm{dec}} \eta \epsilon_{\nu}^{\alpha-1} E_{\mathrm{HN}} \int_{\varepsilon_{\nu, 1}}^{\varepsilon_{\nu, 2}} A_{\exp }\left(\varepsilon_{\nu}\right) \varepsilon_{\nu}^{-\alpha} d \varepsilon_{\nu} \\
& \times \frac{c}{H_{0}} \int_{0}^{z} d z \frac{R_{\mathrm{HN}}(z)}{(1+z)^{\alpha} \sqrt{\Omega_{\mathrm{M}}(1+z)^{3}+\Omega_{\Lambda}}},
\end{aligned}
$$

where $A_{\exp }\left(\varepsilon_{\nu}\right)$, varying with the energy of neutrinos, is the exposure coefficient of the detector for the diffuse neutrinos, with the unit of $\mathrm{cm}^{2} \mathrm{~s}$ sr for $\alpha>2$. While for $\alpha=2$ we have

$$
\begin{aligned}
N_{\nu}= & A^{*} \epsilon_{\mathrm{dec}}^{\prime *} \eta \epsilon_{\nu} E_{\mathrm{HN}} \int_{\varepsilon_{\nu, 1}}^{\varepsilon_{\nu, 2}} A_{\exp }\left(\varepsilon_{\nu}\right) \varepsilon_{\nu}^{-2} d \varepsilon_{\nu} \\
& \times \frac{c}{H_{0}} \int_{0}^{z} d z \frac{R_{\mathrm{HN}}(z)}{(1+z)^{2} \sqrt{\Omega_{\mathrm{M}}(1+z)^{3}+\Omega_{\Lambda}}} .
\end{aligned}
$$

Considering the most sensitive neutrino detector nowadays, i.e., the completed 86 strings IceCube observatory, adopting the effective area varying with the energy of protons, equation (16) gives $N_{\nu}=0.1$ for one year observation.

\section{CONCLUSION AND DISCUSSION.}

ULIRGs are a group of galaxies with ultra-luminous Infrared emission $\left(L_{\mathrm{IR}}>10^{12} L_{\odot}\right)$ and a high SFR $\left(\gtrsim 200 M_{\odot} \mathrm{yr}^{-1}\right)$. Consequently, a high hypernovae rate, $R_{\mathrm{HN}} \sim 1000 \mathrm{yr}^{-1} \mathrm{Gpc}^{-3}$ at redshift $1.2 \lesssim z \lesssim 2.3$ is expected. Since the hypernovae can drive energetic shocks and are able to accelerate protons to $10^{17} \mathrm{eV}$, we propose that huge amounts of protons with spectrum $\frac{d N_{p}^{\prime}}{d \varepsilon_{p}^{\prime}} \propto \varepsilon_{p}^{\prime-\alpha}$ are ejected into these ULIRGs. The observations indicate that ULIRGs have a very high gas surface density, therefore the protons is expected to lose most of their energy through interacting with the dense ISM in ULIRGs before escaping, providing a un-negligible contribution to the PeV neutrinos flux $\left(\sim 2 \times 10^{-9} \mathrm{GeV} \mathrm{cm}^{-2} \mathrm{~s}^{-1} \mathrm{sr}^{-1}\right)$. Its flux comparing with that of the atmosphere neutrinos, the GRB neutrinos and the AGN neutrinos have been presented in Fig.1. The ULIRG neutrino component is likely characterized by a cutoff (or break) at $\sim$ a few $\mathrm{PeV}$ since the hypernovae are likely only able to accelerate the CR protons up to $\sim 100 \mathrm{PeV}$ and the ULIRGs can not confine the protons with energy much larger than $\sim 100$ $\mathrm{PeV}$, either. Such a component may be detected in 20 years by the IceCube full configuration.

Since the diffuse neutrino emission from ULIRGs are expected to be much rarer (see Fig.1), finally we suggest 


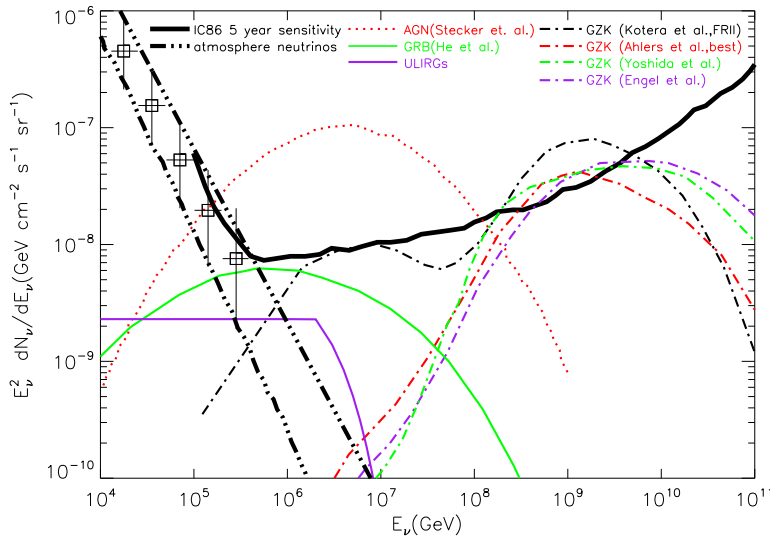

FIG. 1: The flux of the diffuse neutrino emission from ULIRGs (purple solid line), GRBs (green solid line [32]), AGNs (red dotted line [18, 33]), assuming that the spectrum of the ejected protons is $d N_{p}^{\prime} / d \varepsilon_{p}^{\prime} \propto \varepsilon_{p}^{\prime-2}$. The black, red, green and purple dash-dotted lines represent the GreisenZatsepin-Kuzmin (GZK) neutrinos [34, 35] referring to the models in 36] (among Faranoff-Riley type II galaxies, i.e., FRII), 37] (with the best parameters that fit the cosmic ray data), [38] and [39], respectively. The black thick solid line represents the sensitivity of IceCube 86 strings for 5 years. The atmospheric neutrinos are presented by the data with error bars, which is measured by IceCube [40]. The two black dash-triple-dotted lines are the upper bound and lower bound of the atmosphere neutrinos extrapolating to the high energy. that the two PeV neutrino candidates reported by IceCube Collaboration [41], if cosmological, may be from other energetic sources, such as AGNs, GRBs (see [42], but see 32, 43]), and cosmogenic neutrinos (see [44], but see [45, 46]). The origins of the reported two PeV neutrinos are highly controversial so far, we anticipate more observations from the IceCube to draw a firm conclusion in the future.

Acknowledgments. HNH thanks Ruoyu Liu for the useful discussion and Shigehiro Nagataki for the useful suggestion. This work was supported in part by 973 Program of China under grants 2009CB824800 and 2013CB837000, National Natural Science of China under grants 11173064 and 11273063, and by China Postdoctoral science foundation under grant 2012M521137. YZF is also supported by the 100 Talents program of Chinese Academy of Sciences and the Foundation for Distinguished Young Scholars of Jiangsu Province, China (No. BK2012047). SML is also supported by the Recruitment Program of Global Experts from the Central Organization Committee.

${ }^{*}$ Corresponding author.

Electric addresses: hnhe@pmo.ac.cn, taowang@nju.edu.cn, liusm@pmo.ac.cn, dmwei@pmo.ac.cn yzfan@pmo.ac.cn,
[1] Hillas, A. M., Journal of Physics G Nuclear Physics, 31, $95(2005)$

[2] Iwamoto, K., et al. , Nature, 395, 672 (1998)

[3] Mazzali, P. A. et al. , Astrophys. J.,572, L61 (2002)

[4] Mazzali, P. A. et al., Astrophys. J., 614, 858 (2004)

[5] Bell, A. R., \& Lucek, S. G., Mon. Not. R. Astron. Soc., 321, 433 (2001)

[6] Dermer C. D., 2001, in Schlickeiser R., ed., Proc. 27th InternationalCosmic Ray Conference. Hamburg, Germany, p. 2039 (arXiv:astro-ph/0012490)

[7] Sveshnikova, L. G., Astron. Astrophys., 409, 799 (2003)

[8] Wang, X.-Y., Razzaque, S., Mészáros, P., \& Dai, Z.-G., Phys. Rev. D, 76, 083009 (2007)

[9] Budnik, R., Katz, B., MacFadyen, A., \& Waxman, E., Astrophys. J., 673, 928 (2008)

[10] Fan, Y. Z., Mon. Not. R. Astron. Soc., 389, 1306 (2008)

[11] Sanders, D. B., Soifer, B. T., Elias, J. H., et al. 1988, Astrophys. J., 325, 74

[12] Sanders, D. B., \& Mirabel, I. F. 1996, Ann. Rev. Astron. Astrophys., 34, 749

[13] Lonsdale, C. J., Farrah, D., \& Smith, H. E., Astrophysics Update 2, 285 (2006)

[14] Downes, D., \& Solomon, P. M., Astrophys. J., 507, 615 (1998)
[15] Pohl, M., Astron. Astrophys., 287, 453 (1994)

[16] Lacki, B. C., Thompson, T. A., Quataert, E., Loeb, A., \& Waxman, E., Astrophys. J., 734, 107 (2011)

[17] Loeb, A., \& Waxman, E., J. Cos. Astropart. Phys., 5, 3 (2006)

[18] Stecker, F. W. 2007, Journal of Physics Conference Series, 60, 215

[19] Becker, J. K., Biermann, P. L., Dreyer, J., \& Kneiske, T. M., arXiv:0901.1775 (2009)

[20] Dale, D. A., \& Helou, G., Astrophys. J., 576, 159 (2002)

[21] Cappellaro, E., Evans, R., \& Turatto, M., Astron. Astrophys., 351, 459 (1999)

[22] Guetta, D., \& Della Valle, M., Astrophys. J., 657, L73 (2007)

[23] Magnelli, B., Elbaz, D., Chary, R. R., et al., Astron. Astrophys., 528, A35 (2011)

[24] Fukugita, M., \& Kawasaki, M., Mon. Not. R. Astron. Soc., 340, L7 (2003)

[25] Gaisser, T. K., Cambridge and New York, Cambridge University Press, 1990, 292 p. (1990)

[26] Condon, J. J., Huang, Z.-P., Yin, Q. F., \& Thuan, T. X., Astrophys. J., 378, 65 (1991)

[27] Kennicutt, R. C., Jr., Astrophys. J., 498, 541(1998)

[28] Soifer, B. T., Neugebauer, G., Matthews, K., et al., As- 
tronomical Journal, 119, 509 (2000)

[29] Solomon, P. M., \& Vanden Bout, P. A., Ann. Rev. Astron. Astrophys., 43, 677 (2005)

[30] Thompson, T. A., Quataert, E., Waxman, E., Murray, N., \& Martin, C. L., Astrophys. J., 645, 186 (2006)

[31] Kelner, S. R., Aharonian, F. A., \& Bugayov, V. V., Phys. Rev. D, 74, 034018 (2006)

[32] He, H.-N., Liu, R.-Y., Wang, X.-Y., et al. 2012, Astrophys. J., 752, 29

[33] Stecker, F. W. 2007, Astroparticle Physics, 26, 398

[34] Greisen, K. 1966, Physical Review Letters, 16, 748

[35] Zatsepin, G. T., \& Kuz'min, V. A. 1966, Soviet Journal of Experimental and Theoretical Physics Letters, 4, 78

[36] Kotera, K., Allard, D., \& Olinto, A. V. 2010, J. Cos. Astropart. Phys., 10, 13

[37] Ahlers, M., Anchordoqui, L. A., Gonzalez-Garcia, M. C., Halzen, F., \& Sarkar, S. 2010, Astroparticle Physics, 34, 106
[38] Yoshida, S., \& Teshima, M. 1993, Progress of Theoretical Physics, 89, 833

[39] Engel, R., Seckel, D., \& Stanev, T. 2001, Phys. Rev. D, 64, 093010

[40] Abbasi, R., Abdou, Y., Abu-Zayyad, T., et al. 2011, Phys. Rev. D, 83, 012001

[41] A. Ishihara, "IceCube: Ultra-High Energy Neutrinos," Talk at Neutrino 2012, Kyoto, Japan, June 2012; slides available at http://neu2012.kek.jp/index.html.(2012)

[42] Cholis, I., \& Hooper, D. 2012, arXiv:1211.1974

[43] Liu, R.-Y., \& Wang, X.-Y. 2012, arXiv:1212.1260

[44] Barger, V., Learned, J., \& Pakvasa, S. 2013, Phys. Rev. $\mathrm{D}, \mathbf{8 7}, 037302$

[45] Bhattacharya, A., Gandhi, R., Rodejohann, W., \& Watanabe, A. 2012, arXiv:1209.2422

[46] Roulet, E., Sigl, G., van Vliet, A., \& Mollerach, S. 2013, J. Cos. Astropart. Phys., 1, 28 